Review

\title{
Survey on Applications of Electronic Nose
}

\author{
Keerthana, S. and B. Santhi \\ School of Computing, Sastra Deemed to Be University, India
}

Article history

Received: 01-08-2019

Revised: 22-08-2019

Accepted: 13-03-2020

Corresponding Author:

Keerthana, S.

School of Computing, Sastra

Deemed to Be University, India

Email: keerthanasivamayil@gmail.com

\begin{abstract}
Food plays a vital role in our daily life. Providing good quality food to consumers is essential. Food quality can be accessed using the Electronic nose. Electronic nose (E-nose) is an instrument for odor analysis. E-nose mimics the human olfaction system. It is widely used in predicting the quality of foodstuffs and detecting the contamination in foods. E-nose can also be used in outdoor monitoring such as air quality monitoring and detect the hazardous odors emitted wastewater treatment plants. Application of Enose is increasing day by day. In this paper, we consolidated the previous works on E-nose. They had applied different machine learning algorithms to construct a model. In most of the works, for the classification of data, they used Support Vector Machine and Linear Discriminant Analysis, which shows higher accuracy when comparing to other algorithms.
\end{abstract}

Keywords: Electronic Nose, Food Quality, Support Vector Machine, Linear Discriminant Analysis, Support Vector Regression

\section{Introduction}

Traditionally, human tester involved detecting the contamination level of fruits, vegetables, dairy products, animal foods based on the appearance, colour and aroma. It is an inefficient method. E-nose is a non-destructive method to test the quality of products. In 1982, Dodd and Persuade introduced E-nose system. It can detect the volatile compounds emitted from various source. E-nose consists of an array of sensors. Sensors are selected based on applications. E-nose responses are collected using microcontrollers like Arduino, Raspberry pi. PCA (Principal Component Analysis) is one of the feature extraction techniques to choose the important feature in Enose data. Machine learning algorithms such as Naive Bayes (NB), Support Vector Machine (SVM), Linear Regression (LR), Logistic Regression, Linear Discriminant Analysis (LDA), Support Vector Regression (SVR), Partial Least Square Regression (PLSR), Artificial Neural Networks (ANN) and K-Nearest Neighbour (KNN) are applied to the datasets and performance are analysed.

The electronic nose has a significant impact on outdoor monitoring. Humans can't work in specific odor analysis like detecting the gases emitted from the wastewater treatment plant and detecting toxic gases in the air. But E-nose provides an efficient approach to outdoor monitoring. Electronic nose was used in predicting the ripening stage of fruit and also detect the quality of fruit. Nowadays, Human disease is detected using E-nose from the breath sample (Goor et al., 2018). E-nose applications are outlined in the Table 1 and hierarchical chart is shown in Fig. 1. The performances of machine learning algorithms are given in Table 2.

\section{Electronic Nose in Outdoor Monitoring}

The electronic nose can be employed in the area where humans can't detect the odor. For example, wastewater treatment plant emits malodorous, which cause serious health issues on humans. Blanco-Rodríguez et al. (2018) Suggested a method for characterizing the hazardous gas emitted from the wastewater treatment plant using electronic-nose. In their experiment, odor samples collected from the six stages of the plant. They performed signal filtering, normalization and feature extraction with the dataset. They established the correlation between Enose response and olfactometry analysis by using Partial Least Square Regression (PLSR).

Nowadays, Air pollution is a significant concern in this world, which has a severe impact on human health. Jasinski et al. (2018) suggested a method for predicting the toxic gases present in the air. They used three types of electronic nose system depending upon the type of sensor used semiconductor sensor, amperometric sensor and third one combination of both sensors. They had collected the data in 1 minute from each electronic nose and applied PLS regression and SVM. They compared the performance of all three systems. They measured the concentration of the four gases carbon monoxide $(\mathrm{CO})$, Nitrogen dioxide $\left(\mathrm{NO}_{2}\right)$, Sulphur dioxide $\left(\mathrm{SO}_{2}\right)$ and ozone $\left(\mathrm{O}_{3}\right)$ Among the three types of Electronic nose, a combination of both sensors provides better results. 


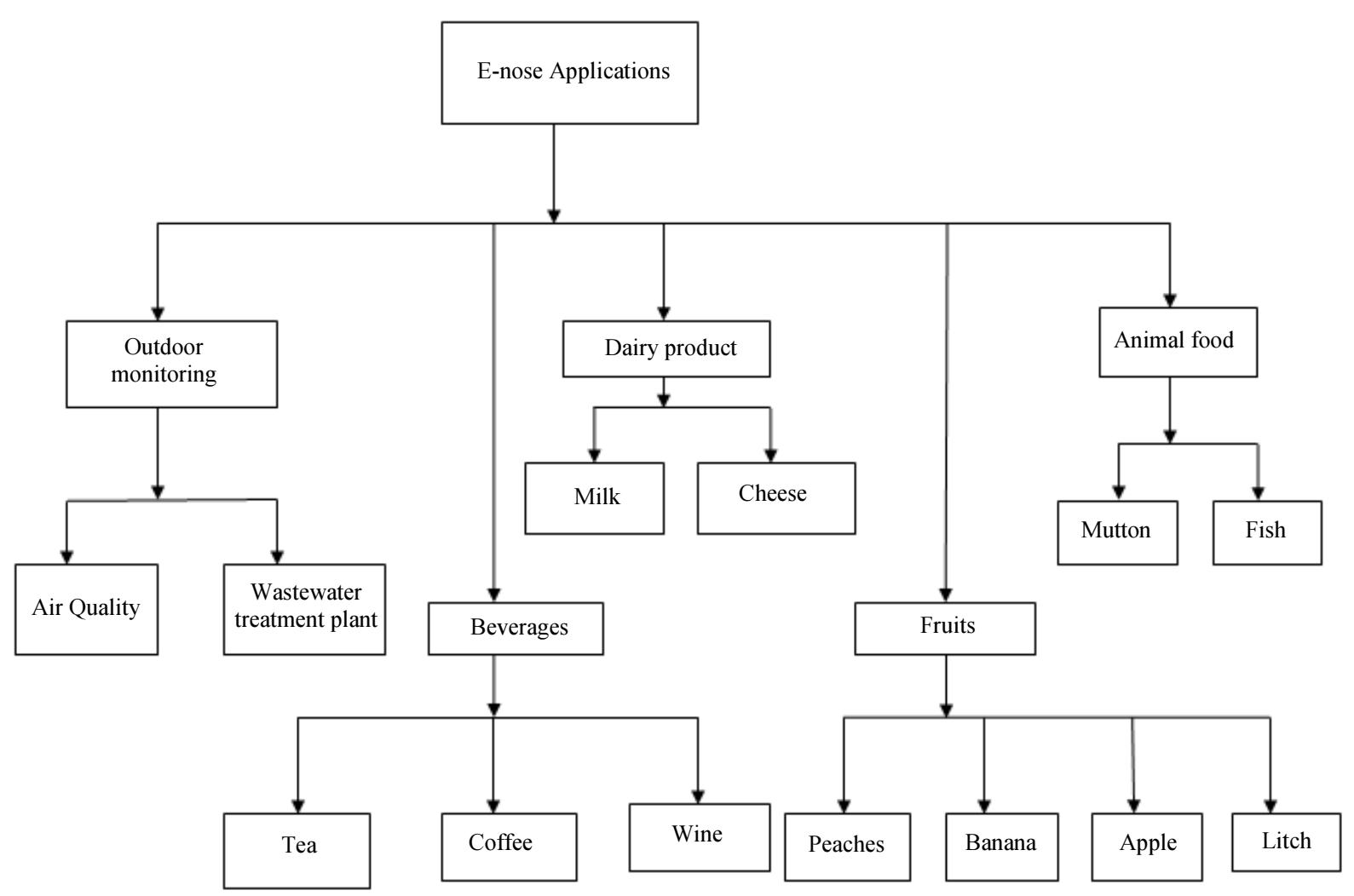

Fig. 1: Hierarchical chart

Table 1: Electronic nose applications

\begin{tabular}{|c|c|c|c|c|}
\hline No & Data & Purpose & E-nose configuration & Reference \\
\hline 1 & $\begin{array}{l}\text { Wastewater } \\
\text { treatment plant }\end{array}$ & $\begin{array}{l}\text { Characterize the hazardous gas } \\
\text { emitted from the wastewater treatment } \\
\text { plant using electronic-nose }\end{array}$ & $\begin{array}{l}\text { TGS2611, TGS2602, TGS2610, } \\
\text { TGS826 and TGS2600 }\end{array}$ & (Blanco-Rodríguez et al., 2018) \\
\hline 2 & Air & Detect toxic gases in the air & $\begin{array}{l}\text { Semiconductor sensors } \\
\text { and amperometric sensors }\end{array}$ & (Jasinski et al., 2018) \\
\hline 3 & Potato & To detect soft rot disease in potato & Warwick OLFaction & (Rutolo et al., 2018) \\
\hline 4 & Apple & $\begin{array}{l}\text { To detect and recognize the } \\
\text { fresh and moldy apple }\end{array}$ & PEN3 & (Jia et al., 2019). \\
\hline 5 & Herbal medicine & $\begin{array}{l}\text { To classify the Chinese } \\
\text { herbal medicine of } 12 \text { types }\end{array}$ & TGS (Taguchi gas sensors) & (Zhan, 2018) \\
\hline 6 & Cherry Tomato & $\begin{array}{l}\text { To detect the quality of cherry tomato } \\
\text { and classify them into four groups }\end{array}$ & $\begin{array}{l}\text { Ammonia, sulphur compounds, } \\
\text { Hydrogen, Organic acid esters, } \\
\text { Sensitive to methane, Aromatics } \\
\text { compounds, Aliphatic } \\
\text { hydrocarbons, Hydrocarbons, } \\
\text { Aromatic compounds, Alcohol } \\
\text { And organic solvents, Alkenes, } \\
\text { aromatic compounds, less polar } \\
\text { compounds }\end{array}$ & (Feng et al., 2018) \\
\hline 7 & Banana & To predict the quality of banana & $\begin{array}{l}\text { MQ-3, MQ-5, M Q-9, MQ-131, } \\
\text { MQ-136, MQ-135 }\end{array}$ & (Sanaeifar et al., 2016) \\
\hline 8 & $\begin{array}{l}\text { Royal delicious } \\
\text { apple }\end{array}$ & $\begin{array}{l}\text { To predicting the quality of } \\
\text { fresh, half and full contaminated } \\
\text { Royal delicious apple. }\end{array}$ & $\begin{array}{l}\text { Ethanol, toluene, xylene, } \\
\text { Ammonia, (Ammonia and toluene), } \\
\text { Alcohol \& organic solvent vapor, } \\
\text { Hydrogen \& carbon monoxide }\end{array}$ & (Rayappan et al., 2018) \\
\hline 9 & Citrus Fruits & $\begin{array}{l}\text { To detect the presence of Bactrocera } \\
\text { dorsalis in citrus fruits }\end{array}$ & $\begin{array}{l}\text { TGS2620, } \\
\text { TGS2610, TGS2600, } \\
\text { TGS2602, TGS2603, } \\
\text { MP901 }\end{array}$ & (Wen et al. 2019) \\
\hline 10 & Litch & $\begin{array}{l}\text { To detect the quality of } \\
\text { litch in various atmosphere }\end{array}$ & PEN3 & (Xu et al., 2016) \\
\hline
\end{tabular}




\begin{tabular}{|c|c|c|c|c|}
\hline 11 & Peaches & $\begin{array}{l}\text { To discriminate and identify the } \\
\text { contamination of fungi in peaches }\end{array}$ & PEN3 & (Liu et al., 2018) \\
\hline 12 & Wine & $\begin{array}{l}\text { To detect the spoilage } \\
\text { threshold of wine }\end{array}$ & $\begin{array}{l}\text { MQ-3, MQ-4, MQ-6 } \\
\text { (two of each type) }\end{array}$ & (Rodriguez et al., 2019) \\
\hline 13 & Tea & $\begin{array}{l}\text { To detect appearance and aroma } \\
\text { quality of Tea using computer } \\
\text { vision and E-nose }\end{array}$ & PEN3 & (Xu et al., 2018) \\
\hline 14 & Coffee & $\begin{array}{l}\text { To classify the coffee samples } \\
\text { using E-nose }\end{array}$ & PEN2 & (Yasuo et al., 2019) \\
\hline 15 & Tea and coffee & $\begin{array}{l}\text { To classify the Tea and coffee } \\
\text { samples }\end{array}$ & $\begin{array}{l}\text { TGS822, TGS830, } \\
\text { TGS825, } \\
\text { TGS821, TGS832, } \\
\text { TGS826, TGS816, } \\
\text { TGS2600, TGS2602, } \\
\text { TGS2610, TGS2611, } \\
\text { TGS2620 }\end{array}$ & (Omatu and Yano, 2016) \\
\hline 16 & Mutton & $\begin{array}{l}\text { To detecting adulteration of mutton } \\
\text { with duck meat using E-nose }\end{array}$ & PEN3 & (Wang et al., 2019) \\
\hline 17 & Fish & $\begin{array}{l}\text { To classify the different } \\
\text { species of fish }\end{array}$ & $\begin{array}{l}\text { TGS } 2610, \text { TGS } 2620 \\
\text { TGS } 830, \text { TGS } 880, \\
\text { TGS } 2104, \text { TGS } 2602 \text {, } \\
\text { TGS } 825, \text { TGS } 826 \text {, }\end{array}$ & (Güney and Atasoy, 2015) \\
\hline 18 & Pecorino cheese & To classify the cheese using E-nose & $\begin{array}{l}\mathrm{SnO}_{2},\left(\mathrm{SnO}_{2}+\mathrm{SiO} 2\right), \\
\left(\mathrm{SnO}_{2}+\mathrm{Au}\right), \\
\left(\mathrm{SnO}_{2}+\mathrm{Ag}\right) \text { and } \\
\left(\mathrm{SnO}_{2}+\mathrm{PD}\right) \text { and } \\
\mathrm{WO} 3 .\end{array}$ & (Cevoli et al. 2011) \\
\hline 19 & Milk & To detect the adulteration in milk & $\begin{array}{l}\text { MQ3, TGS2620 SP3- } \\
\text { AQ2, MQ136, TGS822, } \\
\text { TGS2602, MQ8, TGS813, }\end{array}$ & (Tohidi et al., 2017) \\
\hline
\end{tabular}

Table 2: Performances of algorithms

\begin{tabular}{|c|c|c|c|}
\hline No & Data & Algorithm & Performance \\
\hline 1 & $\begin{array}{l}\text { Toxic gases from the } \\
\text { wastewater treatment plant }\end{array}$ & PLSR & $\begin{array}{l}\text { PLSR with R-square was } 0.9967 \text { and Root Mean Square } \\
\text { Error (RMSE) } 1.17 \times 10^{4}\end{array}$ \\
\hline 2 & Air & $\begin{array}{l}\text { Support Vector Regression (SVR) } \\
\text { and PLSR }\end{array}$ & SVR provides lower RMSE than PLSR \\
\hline 3 & Potato & $\begin{array}{l}\text { LDA, SVM, NB, Radial Basis } \\
\text { Ensemble }\end{array}$ & $\begin{array}{l}\text { SVM, LDA and Radial Basis Ensemble shows higher } \\
\text { accuracy of } 100 \%\end{array}$ \\
\hline 4 & Moldy Apple & LDA, SVM, BPNN, RBFNN, & $\begin{array}{l}\text { BPNN shows higher accuracy with } 90 \% \text { and } 72 \% \text { for } \\
\text { group A and group B }\end{array}$ \\
\hline 5 & Herbal medicine & LDA, SVM, DT, KNN, NB, BP & $\begin{array}{l}\text { SVM and LDA shows accuracy with } 98.94 \% \text { and } \\
98.34 \%\end{array}$ \\
\hline 6 & Cherry Tomato & $\begin{array}{l}\text { Single Feed Forward Neural } \\
\text { Network, PLS }\end{array}$ & $\begin{array}{l}\text { Single Feed Forward Neural Network shows higher with } \\
\text { Higher } \mathrm{R}^{2} \text { and lower RMSE than PLS }\end{array}$ \\
\hline 7 & Banana & $\begin{array}{l}\text { PLS, Multiple Linear Regression } \\
\text { (MLR) and Support Vector } \\
\text { Regression (SVR) }\end{array}$ & $\begin{array}{l}\text { SVR outperforms the MLR with Higher R and lower } \\
\text { RMSE }\end{array}$ \\
\hline 8 & Royal delicious apple & $\begin{array}{l}\text { PCA and wards method of } \\
\text { hierarchical cluster analysis }\end{array}$ & Both established correlations between samples of apple \\
\hline 9 & Citrus Fruits & LDA & LDA show accuracy with $98.21 \%$. \\
\hline 10 & Litch & LDA, BPNN, BPNN-PLSR, CCA, & $\begin{array}{l}\text { BPNN-PLSR shows better accuracy than other } \\
\text { algorithms }\end{array}$ \\
\hline 11 & Peach & PLS-DA & It shows higher prediction rates \\
\hline 12 & Tea & $\begin{array}{l}\text { KNN, Multinomial Logistic } \\
\text { Regression (MLR), SVM }\end{array}$ & SVM shows higher prediction rate with $100 \%$ accuracy \\
\hline 13 & Coffee & $\begin{array}{l}\text { Common Dimension Analysis } \\
\text { (ComDim) and LDA }\end{array}$ & LDA provides $100 \%$ accuracy \\
\hline 14 & Tea and Coffee & $\begin{array}{l}\text { Learning Vector } \\
\text { Quantization (LVQ) }\end{array}$ & $\begin{array}{l}\text { LVQ shows } 96 \% \text { accuracy in four kinds of Tea and } 89 \% \\
\text { in five kinds of coffee }\end{array}$ \\
\hline 15 & Mutton & $\begin{array}{l}\text { Linear regressions, Fisher Linear } \\
\text { Discriminant Analysis (FLDA), } \\
\text { and Multilayer Perceptron } \\
\text { Neural Networks analysis MLPN }\end{array}$ & $\begin{array}{l}\text { (MLPN) FLDA and patterns shows accuracy } \\
98.2 \% \text { and } 96.5 \%\end{array}$ \\
\hline 16 & Fish & NB, KNN and LDA & $\begin{array}{l}\text { Accuracy of NB, KNN and LDA are } 84.73,80 \text { and } \\
82.4 \text {. NB shows maximum accuracy }\end{array}$ \\
\hline 17 & Pecorino cheese & Multi-Layer Perceptron (MLP) & MLP correctly classified the cheese \\
\hline 18 & Milk & LDA, SVM & $\begin{array}{l}\text { SVM showed accuracy values of } 94.64,92.85 \text { and } \\
87.75 \% \text { for formalin, hydrogen peroxide and sodium } \\
\text { hypochlorite, respectively. }\end{array}$ \\
\hline
\end{tabular}


Feature extraction techniques were applied to the datasets to select critical features. Most commonly used feature extraction is PCA.

Kong et al. (2019) Proposed a new procedure for feature extraction Weighted Summation (WS). Gaseous pollutants emitted from the pig farm can affect the environment and also a severe impact on the health of humans. They had collected data from pig farm data using E-nose. And they had applied Weighted Summation to datasets. They compared the results with existing feature extraction algorithms and weighted summation showed higher accuracy.

Herrero et al. (2016), had proposed classification of water pollutants using wireless portable electronic noses.

\section{Disease Detection Using Electronic-Nose}

Detection of soft rot disease in potato (Rutolo et al., 2018). In this work, they used WOLF 4.1 (Warwick OLFaction), electronic nose for predicting the contamination of Pectobacterium carotovorum in potato. Data analyzed using algorithms such as LDA, SVM, Naive Bayes, ensemble methods.

Jia et al. (2019) suggested PEN3 was used to detect the level of contamination of moldy apple inoculated with Penicillium expansum and Aspergillus niger. Dataset collected from both the apple inoculated with and without bacteria. Four machine learning algorithms were used to analyze the data such as Back Propagation Neural Network (BPNN), SVM and radial basis function neural network (RBFNN), Linear Discriminant Analysis (LDA). They found that the BPNN shows higher accuracy among all the algorithms.

Electronic nose not only detects the human disease but also recognizes the condition in plant and animal (Wilson, 2018). Electronic nose detects the disease based on the Volatile Compound (VOC) emitted from the sample.

\section{Discrimination of Substance Using Electronic Nose}

Zhan (2018) suggested a method for discriminating 12 different categories of Chinese herbal medicine using electronic nose. In this work, the electronic nose consists of 16 TGS (Taguchi gas sensors) made in Japan. Data acquired from 600 samples one by one. And they preprocessed the dataset and applied machine learning algorithm such as Support Vector Machine (SVM), Decision Tree (DT), Naive Bayes (NB), Conformal Prediction K-Nearest Neighbour (CP-KNN), Artificial Neural Network (ANN), Linear Discriminant Analysis (LDA). Among them, SVM and LDA show higher accuracy with $98.94 \%$ and $98.33 \%$. But the KNN (CP-1NN and $\mathrm{CP}-3 \mathrm{NN}$ ) provides the prediction reliability.

Centonze et al. (2019) in their paper, they used electronic nose to discriminate the different varieties of oranges belongs to three regions Italy, South Africa and Spain. They applied multivariate statistical models to the E-nose response. And the LDA provides better prediction accuracy.

\section{Fruits and Vegetables Quality Prediction Using Electronic Nose}

The freshness of cherry tomato was evaluated in their work (Feng et al., 2018). They divided tomatoes into two groups. Two groups of cherry tomato treated with and without high-pressure Argon in the ratio of 0.4, 0.8 and 1.2 Mpa. They used Argon gas as a preservative. And data collected from both groups of tomato. After data acquisition, they used Partial Least Square Regression (PLSR) and Single Layer Feed Forward Neural Network. And they made the comparative study between the two algorithms. Based on the E-nose data, they classified the cherry tomato into four groups.

Jia et al. (2019) they inoculated Golden delicious apples with Penicillium expansum and Aspergillus niger. The PEN3 was used to identify the fresh and moldy apples (apples inoculated with Penicillium expansum and Aspergillus Niger). Gas emitted from apple matches with sensors available in PEN3. E-nose response was analyzed using LDA, BPNN, SVM, Radial Basis Function Neural Network (RBFNN). BPNN provided the best accuracy among all methods. E-nose identifies not only fresh apple and but also discriminated moldy apples inoculated with Penicillium expansum and Aspergillus niger.

Brezmes et al. (2000) suggested a method for monitoring fruit ripeness using E-nose. For this purpose, they used Peach, Apple and Pear. They observed the fruit from the day of harvest until it became overripe. They used the neural network to classify the fruits into different stages of green, ripe and overripe. Peach and Pear shows higher accuracy than Apple.

Rayappan et al. (2018) proposed a method for predicting the quality of fresh and contaminated Royal delicious apple. E-nose consists of six readymade sensors and integrated into a single circuit. E-nose values recorded at the sample time of 1s. They applied PCA and the ward's method of cluster analysis to data. This method found a correlation between the different stages of apple.

Wen et al. (2019) sweeping E-nose was used to identify and detect the presence of Bactrocera dorsali in citrus fruits. They applied PCA and LDA to the E-nose data. They found that LDA provides better performance with an accuracy of $98.21 \%$ and discriminate the different stages of incubation and invasion.

$\mathrm{Xu}$ et al. (2016) used PEN3 E-nose predicts to litch quality. Litch quality was detected in different environments (normal temperature, refrigerator, controlled condition). The hardness of the litch sample was obtained from E-nose. LDA, Canonical Correlation Analysis (CCA), BPNN and BPNN- Partial Least Squares Regression (BPNN-PLSR), were employed to sensor data. They found that BPNN-PLSR effectively predicted hardness of litchi under refrigerator storage conditions and 
a controlled-atmosphere environment, but it was poor in normal storage.

Liu et al. (2018) proposed method for identifying fungal contamination in peaches using a PEN3. Peaches were inoculated with spoilage fungi such as Botrytis cinerea, Monilinia fructicola and Rhizopus stolonifer and then stored for long periods. E-nose was used to analyze volatile compounds generated in the fungi-inoculated peaches. Data pre-processing was done by Standard Normal Variate(SNV) to eliminate the signal drift. PLSR was applied to classify the fungi species. They successfully discriminated Peach sample inoculated with fungi after 48hours of storage. The statistical results showed Volatile compounds of peach was affected by the total count and species of fungi.

Sanaeifar et al. (2016) suggested E-nose for predicting the properties of banana. They made the comparison between E-nose response data and quality indices of banana was applying by Partial Least Square, Multiple Linear Regression and Support Vector Regression. They found $\mathrm{pH}$ and Titratable Acidity of quality indices of banana showed poor correlation with E-nose response.

They found that the quality indices of banana predicted using SVR were better than other algorithms. They discovered that E-nose was reliable to predict the properties of banana.

\section{Quality Prediction of Beverages}

Electronic nose provides an efficient method for checking the quality of beverages. (Rodriguez et al. 2019) Suggested design for identifying wine quality is analyzed using the electronic nose. E-nose response of wine collected at the sampling frequency of $18.5 \mathrm{~Hz}$ during 180 seconds. And they classified the dataset into high quality, average quality and low quality. They identified the threshold of wine quality.

$\mathrm{Xu}$ et al. (2018) Proposed a method for predicting the quality of Tea by E-nose and Computer Vision System (CVS). E-nose was used to categorize the quality of Tea based on the aroma. CVS analyzed the appearance of the Tea; it captures the image of Tea and extracts information such as size and color. They made a comparison between E-nose signals and CVS signals. They developed the data fusion strategy combining both the methods of E-nose and CVS to predict the quality of the E-nose.

Yasuo et al. (2019) proposed a method to analyze the coffee sample of six types using PEN2 (seven MOS sensors). Common dimension analysis was used to reduce the large datasets and LDA was applied to classify the samples. This method is efficient to classify the coffee samples.

Omatu and Yano (2016) Designed the E-nose (14 sensors) system to discriminate Tea or coffee based on aroma emitted from the samples of different concentrations. They used the Learning Vector
Quantization neural network to analyze the data. After reducing the E-nose noise, obtain the maximum value of odor. Normalize the datasets; values were affected due to different concentration level. E-nose, along with learning vector quantization neural network was efficient to discriminate between Tea or coffee.

\section{Electronic Nose in Animal Food Analysis}

Animal foods are highly perishable. Electronic nose used to detect the quality and identify the adulteration of animal food. In their study, (Wang, 2019) suggested a method for detecting adulteration of mutton with duck meat using E-nose. They performed Multivariate data analysis by using linear regression, Fisher Linear Discriminant Analysis (FLDA) and Multilayer Perceptron Neural Networks analysis (MLPN) on E-nose signals.

Güney and Atasoy (2015) designed the E-nose (8 sensors) to discriminate between different species of fish. After data acquisition, data pre-processing was done by signal pre-processing, normalization and feature extraction. The proposed Hybrid algorithm shows higher accuracy when compared to all methods KNN, NB, LDA.

\section{Electronic Nose for Edible Oil}

Nowadays, E-nose can also be used in detecting the quality of the oil. (Majchrzak et al., 2017) proposed method for determination of the product's geographical origin and further in the detection of adulteration as well as deterioration caused by external factors. E-nose used to discriminate between non-oxidized and oxidized oils. They used Cluster Analysis (CA), PCA and LDA to E-nose data. LDA produced better results than CA and PCA in discriminating between oxidized oil and non-oxidized oil.

Upadhyay et al. (2017) designed the E-nose (18 Metal Oxide Semiconductor sensors) used for monitoring the disposal time of deep-fried sunflower oil stabilized with natural oxidants.

Rapeseed is one of the sources of edible oil (Gancarz et al., 2017) agrinose used for detecting the quality of rapeseed. Agrinose(eight MOS sensors), sensors were selected based on lower power consumption, low susceptibility to humidity and temperature. The quality of rapeseed was detected using Enose during 31 days of storage was studied. Agrinose used for the examination of Colony Forming Unit, Ergosterol content, Fourier Transform Infrared Spectroscopy and Volatile Organic Compounds. Agrinose monitored the microbiological count of rapeseed during the first twelve days of storage. PCA had shown a correlation between Ergosterol content, sensor response, Colony Forming Unit and the type of microflora.

\section{Electronic Nose in Dairy Product}

E-nose provides the best quality assessment of the dairy product. It is used to detect the adulteration in milk and classify the cheese according to manufacturing techniques. 
Cevoli et al. (2011) proposed E-nose to classify the pecorino cheese. They used ANN and E-nose data feed as input to the ANN. After feature extraction using PCA, feed the features to the ANN. They made a comparison between the before and after feature extraction. Before feature extraction, it showed higher accuracy. Feature extraction was not efficient in this method.

Tohidi et al. (2017) suggested a technique using the electronic nose to detect adulteration in raw milk. After data acquisition, data pre-processing involves steps such as baseline correction, compression and normalization. PCA was used to reduce the dimensionality of data. They used multivariate data analysis such as LDA, SVM to analyze E-nose. SVM showed higher accuracy. This method found the adulteration and percentage of adulteration by using chemometrics.

\section{Conclusion}

In this review, we have given a summary of E-nose applications in various fields, finding the adulteration in mutton, milk and predicting the quality of fruits. It can also classify the food based on the aroma emitted from food. Most of the classification techniques provide more than $90 \%$ accuracy. Among them, SVM and LDA provide $100 \%$ prediction rate and Support Vector Regression provides lower RMSE and higher $\mathrm{R}^{2}$. BPNN delivers the desired performance in most of the cases.

\section{Acknowledgement}

We wish to express our sincere thanks to Sastra Deemed To be University for providing facilities.

\section{Author's Contributions}

Literature review and drafting was done by B. Santhi and Manuscript was written by S. Keerthana.

\section{Ethics}

There is no ethical issues in publishing the paper.

\section{References}

Blanco-Rodríguez, A., V.F. Camara, F. Campo and H. Melo, A.R. Garcia-ramirez et al., 2018. Development of an electronic nose to characterize Odours emitted from different stages in a wastewater treatment plant. Water Res., 134: 92-100. DOI: 10.1016/j.watres.2018.01.067

Brezmes, J., E. Llobet, X. Vilanova, G. Saiz and X. Correig, 2000. fruit ripeness monitoring using an electronic nose. Sensors Actuators B: Chemical, 69: 223-29. DOI: 10.1016/S0925-4005(00)00494-9
Centonze, V., V. Lippolisb, S. Cervellierib, A. Damascellib and G. Casielloa, et al., 2019. Discrimination of Geographical Origin of Oranges (Citrus Sinensis L. Osbeck) by Mass SpectrometryBased Electronic Nose and Characterization of Volatile Compounds. Food Chem., 277: 25-30. DOI: 10.1016/J.FOODCHEM.2018.10.105

Cevoli, C., L. Cerretaniab, A. Gorib, M.F. Cabonib and T. Gallina et al., 2011. Classification of pecorino cheeses using electronic nose combined with artificial neural network and comparison with GCMS analysis of volatile compounds. Food Chem., 129: $1315-19$.

DOI: 10.1016/J.FOODCHEM.2011.05.126

Feng, L., M. Zhang, B. Bhandari and Z. Guo, 2018. A novel method using MOS electronic nose and elm for predicting postharvest quality of cherry tomato fruit treated with high pressure argon. Comput. Electronics Agric., 154: 411-19. DOI: 10.1016/J.COMPAG.2018.09.032

Gancarz, M., J. Wawrzyniak, M. Gawrysiak-Witulska, D. Wiącek and A. Nawrocka et al., 2017. Application of electronic nose with MOS sensors to prediction of rapeseed quality. Measurement, 103: 227-234. DOI: 10.1016/j.measurement.2017.02.042

Goor, R.V.D., M.V. Hooren, A.M. Dingemans, B. Kremer and K. Kross et al., 2018. Training and validating a portable electronic nose for lung cancer screening. J. Thoracic Oncol., 13: 676-681. DOI: $10.1016 /$ j.jtho.2018.01.024

Güney, S. and A. Atasoy, 2015. Study of fish species discrimination via electronic nose. Comput. Electronics Agric., 119: 83-91. DOI: 10.1016/j.compag.2015.10.005

Herrero, L., J. Lozano, P. Santos and I. Su, 2016. Online classification of pollutants in water using wireless portable electronic noses. Chemosphere, 152: $107-116$.

DOI: 10.1016/j.chemosphere.2016.02.106

Jasinski, G., L. Wozniak, P. Kalinowski and P. Jasinski, 2018. Evaluation of the electronic nose used for monitoring environmental pollution. Proceedings of the International Scientific Conference Optoelectronic Electronic Sensors, Jun. 17-20, IEEE Xplore press, Warsaw, Poland, pp: 1-4. DOI: $10.1109 /$ COE.2018.8435146

Jia, W., G. Liang, H. Tian, J. Sun and C. Wan, 2019. Electronic nose-based technique for rapid detection and recognition of moldy apples. DOI: $10.3390 / \mathrm{s} 19071526$

Kong, C., S. Zhao, X. Weng, C. Liu and R. Guan et al., 2019. Weighted summation: Feature extraction of farm pigsty data for electronic nose. IEEE Access, 7: $96732-96742$. DOI: 10.1109/ACCESS.2019.2929526 
Liu, Q., N. Zhao, D. Zhou, Y. Sun and K. Sun et al., 2018. Discrimination and growth tracking of fungi contamination in peaches using electronic nose. Food Chem., 262: 226-34. DOI: 10.1016/j.foodchem.2018.04.100

Majchrzak, T., W. Wojnowski, T. Dymerski, J. Gębicki and J. Namieśnik, 2017. Electronic noses in classification and quality control of edible Oils: A Review. Food Chem., 246: 192-201. DOI: 10.1016/J.FOODCHEM.2017.11.013

Omatu, S. and M. Yano, 2016. E-nose system by using neural networks, Neurocomputing, 172: 394-98. DOI: 10.1016/J.NEUCOM.2015.03.101

Rayappan, J., N. Nesakumar, K.J. Babu, C.S. Srinandan and J.B. Balaguru Rayappan, 2018. An electronic nose for royal delicious apple quality assessment - a tri-layer approach. Food Res. Int., 109: 44-51. DOI: 10.1016/J.FOODRES.2018.04.009

Rodriguez, J.C., E.S. Albarracin, A.J. da Silva and T.A.E. Ferreira, 2019. Electronic nose dataset for detection of wine spoilage thresholds. Data Brief, 25: 104-202. DOI: 10.1016/J.DIB.2019.104202

Rutolo, M.F, J.P, Clarkson and J.A.Covington, 2018. The use of an electronic nose to detect early signs of soft-rot infection in potatoes. Biosystems Eng., 167: 137-43.

DOI: 10.1016/J.BIOSYSTEMSENG.2018.01.001

Sanaeifar, A., S.S. Mohtasebi, M. GhasemiVarnamkhasti and H. Ahmadi, 2016. Application of MOS based electronic nose for the prediction of banana quality properties. Measurement, 82: 105-14. DOI: 10.1016/J.MEASUREMENT.2015.12.041

Tohidi, M., M. Ghasemi-Varnamkhasti, V. Ghafarinia, M. Bonyadian and S. Saeid Mohtasebi, 2017. Development of a metal oxide semiconductor-based artificial nose as a fast, reliable and non-expensive analytical technique for aroma profiling of milk adulteration. Int. Dairy J., 77: 38-46.

DOI: 10.1016/J.IDAIRYJ.2017.09.003
Upadhyay, R., S. Sneha and N.M. Hari, 2017. Lwt-food science and technology frying disposal time of sun $\mathrm{fl}$ ower oil using hybrid electronic nose-fuzzy logic approach. LWT-Food Sci. Technol., 78: 332-39. DOI: 10.1016/J.LWT.2017.01.001

Wang, Q., 2019. College of Food Science and Engineering.

Wen, T., L. Zheng, S. Dong, Z. Gong and M. Sang et al. 2019. Rapid detection and classification of citrus fruits infestation by Bactrocera dorsalis (Hendel) based on electronic nose. Postharvest Biol. Technol., 147: $156-65$ DOI: 10.1016/J.POSTHARVBIO.2018.09.017

Wilson, A.D., 2018. Applications of electronic-nose technologies for noninvasive early detection of plant, animal and human diseases. Chemosensors, 6: 45-45. DOI: 10.3390/chemosensors6040045

Xu, M., J. Wang and S. Gu, 2018. Rapid identification of tea quality by e-nose and computer vision combining with a synergetic data fusion strategy. J. Food Eng., 241: 10-17. DOI: 10.1016/j.jfoodeng.2018.07.020

Xu, S., E. Lü, H. Lu, Z. Zhou and Y. Wang et al., 2016. Quality detection of litchi stored in different environments using an electronic nose. Sensors (Switzerland), 16: 852. DOI: 10.3390/s16060852

Yasuo, G., F. Makimori and E. Bona, 2019. Commercial instant coffee classification using an electronic nose in tandem with the comdim-lda approach.

Zhan, X., X. Guan, R. Wu, Z. Wang and Y. Wang et al., 2018. Discrimination between alternative herbal medicines from different categories with the electronic nose. Sensors, 18: 2936.

DOI: $10.3390 / \mathrm{s} 18092936$ 